\title{
Depresión e ideación suicida en mujeres víctimas de violencia de pareja
}

\author{
Depression and suicide ideation in women \\ victims of intimate partner violence
}

\section{Depressão e ideação do suicídio em mulheres vítimas de violência do parceiro íntimo}

Sebastián Llosa Martínez ORCID ID: 0000-0002-4984-1666 Universidad de la República, Uruguay

Alicia Canetti Wasser ORCID ID: 0000-0002-2667-0876 Universidad de la República, Uruguay

Autor referente: seballosam@gmail.com

Historia editorial: Recibido: 06/02/2017 Aceptado: 03/04/2018

\section{RESUMEN}

La violencia doméstica contra la mujer es un problema de derechos humanos y de salud pública que afecta entre el 15 y el $71 \%$ de las mujeres en el mundo (Organización Mundial de la Salud, 2005). Uruguay tiene una prevalencia de $27,7 \%$ de violencia doméstica contra la mujer, predominando la violencia de pareja, pero no se han estudiado sus efectos en la salud mental con método clínico. Objetivo: cuantificar la frecuencia de depresión e ideación suicida (IS) en mujeres víctimas de violencia de pareja

y estimar la asociación con variables sociodemográficas, momento de la violencia y embarazo, excluyendo otras causas de síntomas depresivos. Método: estudio caso-control con usuarias del Centro de Salud Jardines del Hipódromo en Montevideo, 30 casos y 28 controles. Se aplicó WAST corto, entrevista clínica, Inventario de Depresión y Escala de Ideación Suicida de Beck. Resultados: 56,7 \% de casos presentan depresión vs $14,3 \%$ de controles; el Odds ratio para depresión en casos vs controles es de 7,846. La 
frecuencia de IS es de $20 \%$ de los casos y $3,6 \%$ en los controles y la de depresión+ideación suicida es el 17\% de los casos. La presencia de depresión e IS es independiente del resto de las variables salvo estado civil $\left(\mathrm{x}^{2}=19,408^{\mathrm{a}}, \mathrm{p}<0,001\right)$. Solteras, separadas y/o divorciadas presentan más casos de depresión comparadas con las casadas o en unión libre: $81 \%$ vs $19 \%$. Conclusiones: la violencia de pareja es un factor de riesgo alto para depresión y para conductas suicidas.

Palabras clave: Depresión; ideación suicida; suicidio; violencia de pareja

\section{ABSTRACT}

Domestic violence against women is a human rights and public health problem that affects between 15 and $71 \%$ of women in the world (World Health Organization, 2005). Uruguay has a prevalence of $27.7 \%$ of domestic violence against women, predominantly intimate partner violence, but its effects on mental health have not been studied with a clinical method. Objective: to quantify the frequency of depression and suicidal ideation (SI) in women victims of intimate partner violence and to estimate the association with sociodemographic variables, moment of violence and pregnancy, excluding other causes of depressive symptoms. Method: case-control study with users from the Centro de Salud Jardines del Hipódromo in Montevideo, 30 cases and 28 controls. We applied short WAST, clinical interview, Depression Inventory and Beck Suicidal Ideation Scale. Results: $56.7 \%$ of cases present depression vs $14.3 \%$ of controls. The Odds ratio for depression in cases vs controls is 7,846 . The frequency of IS is $20 \%$ of the cases and $3.6 \%$ in the controls and that of depression + suicidal ideation is $17 \%$ of the cases. The presence of depression and IS is independent of the rest of the variables except marital status $(x 2=19,408 a, p$ $<0,001)$. Single, separated and / or divorced women have more cases of depression compared to married or in free union: $81 \%$ vs $19 \%$. Conclusions: intimate partner violence is a high risk factor for depression and suicidal behaviors.

Keywords: Depression; suicidal ideation; suicide; intimate partner violence

\section{RESUMO}

A violência doméstica contra as mulheres é um problema de direitos humanos e saúde pública que afeta entre 15 e $71 \%$ das mulheres no mundo (World Health Organization, 2005). O Uruguai tem uma prevalência de $27,7 \%$ de violência doméstica contra mulheres, predominantemente com violência por parceiro íntimo, mas seus efeitos sobre a saúde mental não foram estudados com um método clínico. Para quantificar a freqüência de depressão e ideação suicida (IS) em mulheres vítimas de violência por parceiro íntimo e estimar a associação com variáveis sociodemográficas, tempo de violência e gravidez, excluindo outras causas de sintomas depresivos. Método: estudo casocontrole com as usuárias do Centro de Salud Jardines del Hipódromo de Montevidéu, 30 casos e 28 controles. Foi aplicado WAST curto, entrevista clínica, Inventário de Depressão e 
Escala de Ideação Suicida de Beck. Resultados: $56,7 \%$ dos casos apresentam depressão versus 14,3\% dos controles; 0 odds ratio para depressão nos casos vs controles é de 7.846. A frequência de IS é de $20 \%$ dos casos e 3,6\% nos controles e de depressão + ideação suicida é de $17 \%$ dos casos. A presença de depressão e
IS é independente do resto das variáveis, exceto estado civil $(x 2=$ 19.408a, $p<0,001)$. Mulheres solteiras, separadas e / ou divorciadas têm mais casos de depressão do que casadas ou em união livre: $81 \%$ contra $19 \%$. Conclusões: a violência por parceiro íntimo é um fator de alto risco para depressão e comportamentos suicidas.

Palavras-chave: Depressão; ideação suicida; suicídio; violência do parceiro

egún la encuesta multipaís de la Organización Mundial de la Salud [OMS] (2005), la violencia doméstica (VD) y de pareja (VP) afecta a entre el $15 \%$ y el $71 \%$ de las mujeres de diferentes culturas, encontrándose diferencias de frecuencias en los distintos contextos. Además de ocasionar una marginación del mercado laboral, de la vida en sociedad, lesionar la integridad personal, la participación en la cultura y otros problemas de índole social, la VD se asocia a otras graves consecuencias a la salud física y mental.

Varios autores en distintas regiones plantearon que la violencia doméstica y de pareja (VP) trae consigo problemas médicos tales como mayor hospitalización, aumento de posibilidades de cáncer, infecciones de transmisión sexual y abortos provocados (Gibbons, 2011; Plazaola-Castaño \& Ruiz Pérez, 2004).

Distintas investigaciones confirman que la VD y la VP también afectan la salud mental (OMS, 2005; Lozano, Salas Rosso \& Paullier, 2011). Se documentaron hallazgos de depresión, trastornos de ansiedad, intentos de autoeliminación, consumo problemático de sustancias psicoactivas, somatizaciones, baja autoestima e ideación suicida, entre las consecuencias más frecuentes (Gibbons, 2011). Asimismo se ha reportado que la violencia de pareja contra la mujer aumenta el riesgo de conductas suicidas de las víctimas (Devries et al., 2013; OMS, 2005). 
Como problema de salud pública y de derechos humanos, el abordaje de la VD y la VP es muy reciente en Uruguay. Existen encuestas sobre la violencia doméstica contra la mujer y la basada en género, como primeros aportes a la descripción del problema desde el punto de vista cuantitativo. Estas encuestas son los primeros estudios sistemáticos de carácter nacional al respecto, precedido por los estudios que Romano (2010) refiere circunscriptos a Montevideo y Canelones. La Encuesta de Prevalencia de Violencia Doméstica realizada en 2013 (Ministerio de Salud Pública [MSP], 2013) halló un 27,7 \% de prevalencia en mujeres mayores de 15 años usuarias de Servicios de Salud públicos y privados en el primer nivel de atención en todo el territorio nacional. De estos casos, más del $60 \%$ corresponden a la violencia de pareja fundamentalmente del varón hacia la mujer. En Montevideo la frecuencia de violencia doméstica contra la mujer es de 28,9\%. Desagregados los datos según tipo de violencia, la frecuencia de violencia psicológica es del 25,3\%, física 7,5\% y sexual 7,0\%. La Violencia de Pareja es dos tercios de los casos de violencia doméstica contra la mujer.

Del mismo modo la Primera encuesta nacional de prevalencia sobre violencia basada en género y generaciones (Inmujeres-MIDES, 2013), aplicada a población general de mujeres mayores de 15 años, plantea un orden similar de frecuencias: psicológica $23,1 \%$, física $2,7 \%$ y sexual $0,8 \%$ cuando se trata de violencia actual.

De los problemas de salud mental que surgen a partir del padecimiento de la violencia de pareja, la depresión se encuentra entre los dos más frecuentes reportados por estudios internacionales. A su vez la depresión es considerada una pandemia y la primera causa de discapacidad (OMS, 2005). Si también se considera la magnitud del problema del suicidio en Uruguay, se señala que desde la década del 90 se constatan aumentos a las ya altas tasas registradas (Canetti, 2017).

Para la Organización Panamericana de la Salud (OPS), (2009) la violencia doméstica, la depresión y el suicidio se encuentran entre las once prioridades para la atención de 
la salud mental en la región. A su vez se plantea como objetivo en relación con estos problemas hacer hincapié en la atención primaria de la salud. Se ha visto que las víctimas de VD y VP concurren reiteradas veces a los servicios de salud del primer nivel sin ser detectado su problema. Incluso siendo detectado, han sido revictimizadas y culpabilizadas, derivándolas sistemáticamente a servicios de Salud Mental u otros servicios específicos sin prestar una adecuada primera respuesta de escucha comprensiva y empática (Gibbons, 2011).

La relación que existe entre la aparición de depresión y de ideación suicida con padecer o haber padecido violencia de pareja es algo aún no cuantificado en Uruguay, donde se comienzan a dar los primeros pasos en investigaciones clínicas sistematizadas con relación a estos problemas. Solo ha sido cuantificada la presencia de intentos de autoeliminación en mujeres víctimas de VP no siguiendo un método clínico sino el registro en las historias clínicas (Lozano, Salas Rosso \& Paullier, 2011). La carencia de información de calidad que existe con relación a los efectos de la violencia doméstica en la salud mental de la mujer que la padece dificulta la creación de estrategias de detección precoz adecuadas a la población específica.

La depresión es un grupo heterogéneo de síndromes y trastornos de diferente presentación y etiología (American Psychiatric Association, 2013; PDM Task Force, 2006), pero existe consenso en que las diferentes manifestaciones de lo depresivo se componen de signos y síntomas del humor, vegetativos, cognitivos y psicomotores.

La ideación suicida es una de las conductas que forma parte del continuum del suicidio que va desde la ideación suicida, el intento de autoeliminación, el para suicidio hasta el suicidio consumado (Canetti, 2017). Es una variedad de pensamientos de diferente gravedad: desde desear dejar de vivir, fantasías autodestructivas, evaluar la posibilidad de quitarse la vida, pensar en la oportunidad, el modo y las consecuencias del suicidio. 
El presente estudio se propuso avanzar en el conocimiento sobre la magnitud de la depresión e ideación suicida en mujeres usuarias de servicios de salud que sufren o han sufrido violencia de pareja y sobre el papel de la VP en la aparición de estos problemas. Se buscó responder a las siguientes preguntas: ¿con qué frecuencia aparecen la ideación suicida y la depresión en mujeres víctimas de violencia de pareja?; ¿qué relación existe entre la aparición de depresión e ideación suicida con padecer o haber padecido VP?, ¿es posible identificar y correlacionar en esta población la ideación suicida y la depresión como consecuencia de la VP?; ¿qué incidencia tiene la VP en la aparición de depresión y de ideación suicida frente a la que tienen otros factores psicosociales? Para ello se plantearon como objetivos específicos cuantificar la frecuencia de depresión e ideación suicida en mujeres usuarias del Centro de Salud Jardines del Hipódromo identificadas como entre las que padecen o han padecido violencia de pareja. También se buscó conocer y analizar la relación entre la aparición de estos problemas y la violencia de pareja sufrida por las usuarias, comparando un grupo de víctimas con una población de control no expuesto a VP. Se intenta con ello dimensionar dos de los problemas de salud mental más frecuentes y de mayor morbilidad-mortalidad relacionados a la violencia de pareja.

\section{Método}

Se realizó un estudio cuantitativo transversal, descriptivo, no experimental y de asociación no causal que buscó conocer la frecuencia de depresión e ideación suicida en casos de violencia de pareja y controles, así como la asociación entre las variables de análisis. 


\section{Participantes}

Criterios de inclusión y exclusión

La muestra se conformó con 58 participantes, 30 casos y 28 controles. Se incluyeron a todas las usuarias desde 15 años de edad que, habiendo firmado el consentimiento, aceptaron contestar el cribado y participar de las demás instancias. Son usuarias que concurrieron a controles y consultas de las policlínicas seleccionadas, entre los meses de setiembre a diciembre de 2014 y de febrero a julio de 2015 en días aleatorios. Se excluyeron aquellas que referían duelos por personas queridas ocurridos en el último año, enfermedades crónicas (diabetes e hipertensión arterial), enfermedades endócrinas y cardíacas, dolores crónicos y/o inhabilitantes y limitaciones funcionales (sordera, hipoacusia, dificultades motoras, etcétera). Se trata de población que acude

al Centro de Salud, de una zona de bajo nivel socioeconómico y cultural. Fueron tomadas de 120 mujeres que pasaron por el proceso de selección (62 fueron excluidas por los criterios de exclusión, no aceptar firmar el consentimiento, no completar la evaluación y por aplicación no válida de la técnica de evaluación psicológica).

Comparados los grupos, se observa homogeneidad entre el grupo de víctimas de violencia de pareja y los controles en relación a situación socioeconómica, ocupación, nivel educativo y edad (Tablas 1 y 2). Hay diferencias significativas en el estado civil, con mayor frecuencia de solteras, separadas y viudas en el grupo de estudio. No se manipuló la captación de participantes que homogeneizaran esta variable ya que se buscaba expresar en la muestra la descripción de la población.

\section{Procedimiento}

Se realizó un cribado de las usuarias que concurrieron sin la compañía de sus parejas a las policlínicas de pediatría, ginecología y medicina familiar del Centro de Salud mediante el WAST corto. Se les informó del alcance del estudio así como su confidencialidad y su carácter de voluntaria. Previo consentimiento informado, se 
realizó una entrevista clínica estructurada para determinar la existencia y tipos de violencia de pareja, la presencia de criterios de exclusión y para la recolección de datos socio-demográficos. Luego se aplicó el Inventario de Depresión de Beck versión argentina de 2006 (BDI II) y la Escala de Ideación Suicida de Beck (EISB). Para esto se utilizaron los consultorios contiguos a los que acudían las usuarias, en días aleatorios.

Tabla 1

Características socio-demográficas y comparación estadística del grupo de estudio (CON VP) y grupo control (SIN VP)

\begin{tabular}{|c|c|c|c|c|c|c|c|c|}
\hline \multirow{2}{*}{$\begin{array}{l}\text { Característica } \\
\text { Socio } \\
\text { demográfica }\end{array}$} & \multirow[t]{2}{*}{ Categoría } & \multicolumn{2}{|c|}{ Con VP } & \multicolumn{2}{|c|}{ Sin VP } & \multicolumn{2}{|c|}{ Total } & \multirow[t]{2}{*}{$p$} \\
\hline & & $\mathrm{N}$ & $\%$ & $N$ & $\%$ & $\mathrm{~N}$ & $\%$ & \\
\hline \multirow{2}{*}{$\begin{array}{l}\text { Estado } \\
\text { Civil }\end{array}$} & \multirow{2}{*}{$\begin{array}{l}\text { Casada, Unión } \\
\text { Libre } \\
\text { Soltera Separada } \\
\text { Divorciada }\end{array}$} & 16 & 53,3 & 26 & 92,9 & 42 & 72 & \multirow[b]{2}{*}{0,001} \\
\hline & & 14 & 46,7 & 2 & 7,1 & 16 & 28 & \\
\hline \multirow{3}{*}{$\begin{array}{l}\text { Nivel } \\
\text { Socio } \\
\text { Económico }\end{array}$} & Bajo & $16^{\mathrm{a}}$ & 57,1 & 12 & 42,9 & 28 & 50 & \multirow{3}{*}{$n s^{* *}$} \\
\hline & Medio Bajo & $9^{\underline{a}}$ & 32,1 & 0 & 0 & 9 & 16 & \\
\hline & Medio & $3^{a}$ & 10,7 & 16 & 57,1 & 19 & 34 & \\
\hline \multirow{5}{*}{$\begin{array}{l}\text { Nivel } \\
\text { Educativo }\end{array}$} & Primaria & 2 & 6,7 & 2 & 7,1 & 4 & 7 & \multirow{5}{*}{ ns } \\
\hline & Incompleta & & & & & & & \\
\hline & $\begin{array}{l}\text { Primaria } \\
\text { Completa }\end{array}$ & 7 & 56,7 & 15 & 53,6 & 32 & 55 & \\
\hline & Ciclo Básico & 17 & 23,3 & 10 & 35,7 & 17 & 29 & \\
\hline & $\begin{array}{l}\text { Secundaria } \\
\text { Terciaria }\end{array}$ & 4 & 13,3 & 1 & 3,6 & 5 & 9 & \\
\hline \multirow{2}{*}{$\begin{array}{l}\text { Embarazo } \\
\text { Actual }\end{array}$} & No & 19 & 63,3 & 25 & 89 & 44 & 75,9 & \multirow{2}{*}{ ns } \\
\hline & Sí & 11 & 36,7 & 3 & 11 & 14 & 24,1 & \\
\hline \multirow[t]{2}{*}{ Ocupación } & Ama de Casa & 19 & 63,3 & 20 & 71,4 & 39 & 67,2 & \multirow[b]{2}{*}{ ns } \\
\hline & Empleada & 11 & 36,7 & 8 & 28,6 & 19 & 32,8 & \\
\hline
\end{tabular}

Nota: a EI n CON VP en la variable Estado Civil tiene dos casos sin registro

${ }^{* *} \mathrm{~ns}=\mathrm{p} \geq 0,05$ 
Tabla 2

Comparación estadística de la edad en el grupo de estudio (Con VP) y grupo control $(\operatorname{Sin} V P)$

\begin{tabular}{lllllllll}
\hline & \multicolumn{2}{c}{ Con VP } & Sin VP & & Total & & $p$ \\
\hline \multirow{2}{*}{ Edad } & Media & DE & Media & DE & Media & DE & \\
& 28,8 & 9,2 & 28,9 & 7 & 28,8 & 1 & $\mathrm{~ns}^{*}$ \\
\hline
\end{tabular}

Nota: $\mathrm{DE}=$ Desvío Estándar

*ns =No Significativo, $p \geq 0,05$

\section{Instrumentos}

\section{Woman Abuse Screening Tool (WAST) corto}

Se trata de un cuestionario con validez probada para distinguir las mujeres maltratadas de las no maltratadas por su pareja. El Woman Abuse Screening Tool de Brown, Lent, Brett, Sas y Pederson (Fogarty \& Brown, 2002) consta de 8 preguntas con tres opciones de respuesta que miden la intensidad de la violencia. La sensibilidad fue del $91,4 \%$, la especificidad del $76,2 \%$, el valor predictivo positivo del $40,2 \%$ y el negativo del 98,1\% (Plazaola-Castaño, Ruiz-Pérez, \& Hernández-Torres, 2008). A la versión corta de dos preguntas le añadimos una tercera para cribar la variable violencia pasada.

\section{El Beck Depression Inventory II, BDI-II (Inventario de Depresión de Beck)}

Es un instrumento ampliamente utilizado con población clínica y general desde hace varias décadas. Es un cuestionario auto-informado de 21 ítems (rango: 0-63 puntos) que valora la presencia e intensidad de los síntomas depresivos según criterios diagnósticos del DSM IV y del DSM 5. Se utilizó la versión argentina de 2006 (Brenlla \& Rodríguez, 2006) por ajustarse lingüísticamente mejor a la población de estudio. Para medir la frecuencia de depresión se tomó un punto de corte de 14 puntos según lo establecido por el Manual de uso del instrumento (Brenlla, \& Rodríguez, 2006) para distinguir la depresión leve a partir de ese punto, lo que permitió crear una variable 
dicotómica: con y sin depresión. Igualmente se empleó el punto de corte $\geq 17$ sugerido por Beck para casos clínicos, con 93\% positivos, $18 \%$ falsos positivos para muestra clínica (Beck, Steer, \& Brown, 2011).

Escala de Ideación Suicida de Beck, (EISB)

Es un instrumento auto-informado de amplia utilización en distintas investigaciones, desde que se creó en 1979 hasta la actualidad. Consta de 21 items que valoran la presencia de conductas suicidas. Ha sido adaptada al español del Uruguay y utilizada en investigaciones recientes (Canetti, Larrobla, Reigia, \& Brenes, 2011). Se empleó un punto de corte de 1 punto para definir presencia o ausencia de ideación suicida.

\section{Entrevista clínica estructurada}

La entrevista recabó los datos sociodemográficos (nivel de estudios, ocupación, estado civil, nivel socioeconómico), determinó la existencia o no de violencia de pareja y sus tipos (psicológica, física, sexual y económica). En caso de violencia, si fue hasta hace un año (pasada) o si ocurrió en los últimos 12 meses (actual), así como la presencia o no de los criterios de exclusión. También se registró en esa instancia la condición de embarazadas.

\section{Análisis de datos}

Para la comparación entre grupos y asociación de variables se usaron pruebas no paramétricas (chi cuadrado, prueba exacta de Fisher y U de Mann Whitney). Para la relación entre las distintas variables se aceptó un valor de significación estadística de $p \leq 0,05$. Para el procesamiento y análisis estadístico se utilizó el programa SPSS.

\section{Resguardos éticos}

La investigación se llevó adelante con todos los resguardos éticos, se informó a las participantes de las características del estudio, y participaron del mismo de forma libre 
y voluntaria. Los autores declaran estar en posesión del consentimiento libre e informado. El estudio contó con aval del Comité de Ética en Investigación de Facultad de Medicina de la Universidad de la República y la autorización de la Dirección del Centro de Salud para realizar las entrevistas y aplicar la metodología prevista. Para los casos de violencia de pareja y/o de riesgo suicida se implementaron estrategias de atención acordes a cada caso, que el propio investigador a cargo de las entrevistas y aplicación de instrumentos inició. Esta atención y seguimiento de casos no forma parte de la investigación sino que responde al compromiso ético con las participantes.

\section{Resultados}

De la muestra de 30 casos y 28 controles, en el grupo de casos los tipos de violencia encontrados muestran las siguientes frecuencias: $90 \%$ presentaban violencia psicológica, $50 \%$ física, $6,7 \%$ sexual y $26,7 \%$ económica. La mayoría habían experimentado la VP en el pasado (71\%) y cerca de la cuarta parte eran víctimas de VP actual (en los últimos 12 meses).

Los resultados se presentan agrupados según variables dependientes (depresión, ideación suicida, depresión+ ideación suicida).

\section{Depresión}

Se comparó la frecuencia de depresión en los casos de violencia con la de los controles aplicando prueba de chi cuadrado/prueba exacta de Fisher y odds ratio. La frecuencia de depresión fue significativamente mayor en el grupo de víctimas de VP en relación al control: el 56,7 \% de los casos de violencia presentan depresión vs 14,3\% de depresión en los controles (Prueba exacta de Fisher $p=0,001$ ) (Tabla 3).

El Odds ratio para depresión en casos vs controles es de 7,846, lo que indica que la probabilidad de tener depresión al menos leve (puntaje BDI II $\geq 14$ ) es de casi ocho veces más en las mujeres expuestas a VP que en los controles. 
Si se toma como punto de corte $\geq 17$, la frecuencia de depresión es de $30 \%$ en casos y $11 \%$ en controles, no siendo la diferencia estadísticamente significativa (prueba exacta de Fisher $p>0,1)$ (Tabla 3).

Igualmente se comparó la presencia de depresión en casos y controles empleando el puntaje bruto total del Inventario de Beck (variable continua). Para ello se usó la prueba no paramétrica $U$ de Mann Whitney (Tabla 3). La diferencia entre ambos grupos resultó estadísticamente significativa ( $U$ de Mann Whitney: $p=0,002)$.

Analizadas las frecuencias de depresión según las distintas variables sociodemográficas estudiadas (educación, estado civil, ocupación, nivel socioeconómico), se halló una asociación significativa con la variable estado civil. Agrupada la muestra total según solteras+separadas+divorciadas vs casadas+unión libre, la frecuencia de depresión fue significativamente mayor en el primer grupo: $81,3 \%$ vs 19\% respectivamente (Chi-cuadrado de Pearson $=19,4, \mathrm{p}<001)$ (Tabla 4). La asociación significativa se mantiene cuando se desagrega la muestra en grupo de estudio y control (prueba exacta de Fisher: $p=0,03$ y 0,01 respectivamente), como se ve en la Tabla 5.

\section{Ideación suicida}

Analizada la frecuencia de casos de ideación suicida empleando una variable dicotomizada (con y sin ideación) según corte en 1 punto o más en la Escala de Ideación suicida de Beck (EISB), se halló que un 20\% de los casos con VP presentan ideación suicida vs un 3,6\% en los controles, tendiendo la diferencia a la significación estadística (Fisher para una cola: $p=0,06$ ). El puntaje promedio del EISB en los casos fue de 1,8 $(\mathrm{DE}=0,8)$ y en el grupo control de 0,04 (DE=0,04), con una tendencia a la significación estadística de la diferencia entre los grupos ( $U$. de Mann Whitney $p=0,05$ ) (Tabla 3). 
Se analizó la asociación entre la variable dicotomizada (presencia o ausencia de depresión e ideación suicida) y las variables ocupación, educación, nivel socioeconómico y estado civil. Sólo esta última presenta asociación estadísticamente significativa con depresión e IS (Prueba exacta de Fisher: $p<0,001$ y $p=0,014$ respectivamente) cuando se analiza la muestra total, con un predominio de ambos fenómenos entre las solteras, divorciadas y viudas (Tabla 4). La significación se mantiene para depresión pero se pierde para IS si se desagrega por grupos (con y sin VP) (Tabla 5). 
Tabla 3

Comparación de frecuencias e intensidad de depresión, ideación suicida $y$ depresión+ideación suicida entre víctimas de violencia de pareja (CON VP) y controles $(S I N$ VP) $(N=58)$

\begin{tabular}{|c|c|c|c|c|c|c|c|c|c|c|c|c|c|}
\hline \multirow[t]{2}{*}{ Síntomas } & \multicolumn{3}{|c|}{ Con VP } & \multicolumn{6}{|c|}{ Sin VP } & \multicolumn{3}{|c|}{ Total } & \multirow{2}{*}{$p$} \\
\hline & $\mathrm{n}$ & $\%$ & $M$ & Mdn & DE & $\mathrm{n}$ & $\%$ & $\mathrm{M}$ & Mdn & DE & $\mathrm{N}$ & $\%$ & \\
\hline \multicolumn{14}{|c|}{$\begin{array}{l}\text { Depresión (BDI-II } \\
\geq 14 \text { ) }\end{array}$} \\
\hline Sin Depresión & 13 & 43 & & & & 24 & 86 & & & & 37 & 64 & $0,001 \mathrm{a}$ \\
\hline Con Depresión & 17 & 57 & & & & 4 & 14 & & & & 21 & 36 & \\
\hline & & & $15^{\star}$ & $14^{*}$ & $1,6^{*}$ & & & $8,8^{\star}$ & $8,5^{\star}$ & $1,2^{*}$ & & & \\
\hline
\end{tabular}

\section{Depresión}

(BDI-II $\geq 17$ )

Sin Depresión

2170

2589

4679

Con Depresión

930

$\begin{array}{ll}3 & 11\end{array}$

$1221 \quad 0,1 \mathrm{a}$

Ideación Suicida

$(E I S B \geq 1)$

Sin Ideación

Suicida

2480

2796

5188

Con Ideación $\quad 6 \quad 20$

Suicida

14

$0,06 \mathrm{a}$

$$
1,8^{* *} 0^{* *} \quad 0,8^{* *} \quad 0,04^{* *} 0^{* *} \quad 0,04^{* *}
$$

Depresión

(BDI-II $\geq 14$ )

+ IS $($ EISB $\geq 1)$

Sin Depresión+IS 2583

$58 \quad 100$

5591

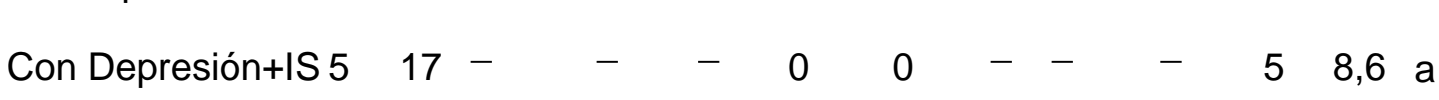

Nota: a Prueba exacta de Fisher

${ }^{*} U$ de Mann Whitney $p=0,002 ;{ }^{*} U$ de Mann Whitney $p=0,05$ 
Tabla 4

Frecuencias de Depresión e Ideación suicida (IS) según estado civil (N=58)

\begin{tabular}{|c|c|c|c|c|c|c|c|}
\hline \multirow[t]{2}{*}{ Síntomas } & \multicolumn{2}{|c|}{$\begin{array}{l}\text { Casada, } \\
\text { Unión Libre }\end{array}$} & \multicolumn{2}{|c|}{$\begin{array}{l}\text { Soltera, Separada, } \\
\text { Divorciada }\end{array}$} & \multicolumn{2}{|c|}{ Total } & \multirow{2}{*}{$p^{\star}$} \\
\hline & $\mathrm{n}$ & $\%$ & $\mathrm{n}$ & $\%$ & $\mathrm{~N}$ & $\%$ & \\
\hline \multicolumn{8}{|l|}{ Depresión (BDI-li $\geq 14$ ) } \\
\hline Sin Depresión & 34 & 81 & 3 & 18,8 & 37 & 63,8 & \multirow[t]{3}{*}{$<0,001$} \\
\hline Con Depresión & 8 & 19 & 13 & 81,3 & 21 & 36,2 & \\
\hline Ideación Suicida (EISB $\geq 1)$ & & & & & & & \\
\hline Sin Ideación Suicida & 40 & 95,2 & 11 & 68,8 & 51 & 88 & \multirow{2}{*}{0,014} \\
\hline Con Ideación Suicida & 2 & 4,8 & 5 & 31,3 & 7 & 12 & \\
\hline
\end{tabular}

*Prueba exacta de Fisher

Del análisis intra-escalar de la EISB se halló una frecuencia de intentos de autoeliminación (IAE) previos autorreportados de $20 \%$ en casos y $11 \%$ en controles. Si bien los IAE entre las víctimas de VP casi duplican a los encontrados en los controles, las diferencias no son estadísticamente significativas (prueba exacta de Fisher: $p=0,5$ ) (Tabla 6). 
Tabla 5

Frecuencias de Depresión e Ideación suicida según estado civil en grupos con y sin violencia de pareja (VP)

\begin{tabular}{|c|c|c|c|c|c|c|}
\hline \multirow{2}{*}{\multicolumn{2}{|c|}{ Síntomas }} & \multicolumn{2}{|c|}{$\begin{array}{l}\text { Casada, } \\
\text { Libre }\end{array}$} & \multicolumn{2}{|c|}{$\begin{array}{l}\text { Soltera, Separada, } \\
\text { Divorciada }\end{array}$} & \multirow[t]{2}{*}{$p^{*}$} \\
\hline & & $\mathrm{n}$ & $\%$ & $\mathrm{n}$ & $\%$ & \\
\hline \multicolumn{7}{|c|}{ Depresión (BDI-li $\geq 14$ ) } \\
\hline \multirow{2}{*}{$\begin{array}{l}\text { Con } \\
\text { VP }\end{array}$} & Sin Depresión & 10 & 62,5 & 3 & 21,4 & \multirow{2}{*}{0,03} \\
\hline & Con Depresión & 6 & 37,5 & 11 & 78,6 & \\
\hline \multirow{2}{*}{ Sin VP } & Sin Depresión & 24 & 92,3 & 0 & 0 & \multirow{2}{*}{0,01} \\
\hline & Con Depresión & 2 & 7,7 & 2 & 100 & \\
\hline \multicolumn{7}{|c|}{ Ideación Suicida (EISB >1) } \\
\hline \multirow{2}{*}{$\begin{array}{l}\text { Con } \\
\text { VP }\end{array}$} & Sin Ideación Suicida & 15 & 93,8 & 9 & 64,3 & \multirow{2}{*}{0,07} \\
\hline & Con Ideación Suicida & 1 & 6,3 & 5 & 35,7 & \\
\hline \multirow[t]{2}{*}{ Sin VP } & Sin Ideación Suicida & 25 & 96,2 & 2 & 100 & \multirow[t]{2}{*}{ ns } \\
\hline & Con Ideación Suicida & 1 & 3,8 & 0 & 0 & \\
\hline
\end{tabular}

${ }^{*}$ Prueba exacta de Fisher ns $=$ no significativo

Tabla 6

Frecuencia de intento de autoeliminación (IAE) previo según grupos con y sin violencia de pareja (VP)

\begin{tabular}{llllllll}
\hline \multirow{2}{*}{ IAE previo } & \multicolumn{2}{l}{ Con VP } & \multicolumn{2}{c}{ Sin VP } & \multicolumn{2}{c}{ Total } & $p^{*}$ \\
& $\mathrm{n}$ & $\%$ & $\mathrm{n}$ & $\%$ & $\mathrm{~N}$ & $\%$ & \\
\hline $\begin{array}{l}\text { Sin IAE Previo } \\
24\end{array}$ & 80 & 25 & 89,3 & 49 & 84,5 & \\
$\begin{array}{l}\text { Con IAE } \\
\text { Previo }\end{array}$ & 6 & 20 & 3 & 10,7 & 9 & 15,5 & \\
\hline
\end{tabular}

*Prueba exacta de Fisher 


\section{Depresión + ideación suicida}

Analizada la frecuencia de Depresión + Ideación Suicida en ambos grupos se halló una frecuencia de $17 \%$ en el grupo de expuestas a VP y ningún caso en los controles (prueba exacta de Fisher $p=0,03)$ (Tabla 3).

Se analizó en el grupo de estudio la asociación de la depresión+ideación suicida con las variables sociodemográficas estudiadas (educación, ocupación, nivel socioeconómico y estado civil) encontrándose una asociación significativa sólo con el estado civil, similar a lo hallado cuando se analiza sólo depresión o sólo ideación suicida (Tabla 7).

Tabla 7

Relación entre Depresión + Ideación Suicida y variables socio-demográficas ( $n=30)$

\begin{tabular}{|c|c|c|c|c|c|c|}
\hline \multirow{3}{*}{ Variables } & \multirow{3}{*}{ Categorías } & \multicolumn{5}{|c|}{ DEPRESIÓN + IDEACIÓN SUICIDA } \\
\hline & & NO & & SI & & $D$ \\
\hline & & $n$ & $\%$ & $n$ & $\%$ & \\
\hline \multirow[t]{2}{*}{ Estado Civil } & Casada, Unión Libre & 15 & 94 & 1 & 6 & \multirow[b]{2}{*}{$0,031^{a}$} \\
\hline & $\begin{array}{l}\text { Soltera, Separada, } \\
\text { Divorciada }\end{array}$ & 8 & 57 & 6 & 43 & \\
\hline \multirow{3}{*}{$\begin{array}{l}\text { Nivel Socio- } \\
\text { económico* }\end{array}$} & Bajo & 12 & 75 & 4 & 25 & \multirow{3}{*}{$\mathrm{ns}^{\mathrm{a}}$} \\
\hline & Medio Bajo & 7 & 78 & 2 & 22 & \\
\hline & Medio & 3 & 100 & 0 & 0 & \\
\hline \multirow[t]{4}{*}{ Educación } & Primaria Incompleta & 2 & 10 & 0 & 0 & \multirow{4}{*}{$\mathrm{ns}^{\mathrm{a}}$} \\
\hline & Primaria Completa & 12 & 71 & 5 & 29 & \\
\hline & Ciclo Básico & 7 & 100 & 0 & 0 & \\
\hline & $\begin{array}{l}\text { Secundaria } \\
\text { Incompleta, Terciario }\end{array}$ & 2 & 50 & 2 & 50 & \\
\hline \multirow[t]{2}{*}{ Ocupación } & Ama De Casa & 14 & 74 & 5 & 26 & \multirow{2}{*}{$\mathrm{ns}^{\mathrm{a}}$} \\
\hline & Empleada & 9 & 82 & 2 & 18 & \\
\hline
\end{tabular}

Nota: dos casos sin dato de Nivel Socio-económico *ns $=$ No significativo $p \geq 0,05$ a prueba exacta de Fisher 


\section{Efectos del embarazo y del momento de exposición a violencia (actual o pasada) sobre depresión e ideación suicida}

Se analizó en la muestra total si estar cursando un embarazo en el momento de la entrevista se relacionaba con la presencia o ausencia de depresión, encontrándose que no hay relación significativa entre ambos fenómenos $\left(x^{2}=0,353, p=0,552\right)$.

Tampoco se encontró asociación entre la presencia o ausencia de depresión, ideación suicida o depresión + ideación suicida en el grupo de estudio con el momento en que se presentó la situación de violencia (actual o pasada), lo que indicaría que la relación entre depresión, ideación suicida y violencia es independiente del tiempo transcurrido desde la exposición a violencia de pareja.

\section{Discusión}

Como se señaló antes, en Uruguay no hay hasta el momento estudios clínicos sobre frecuencia o prevalencia de problemas de salud mental vinculados a la violencia doméstica o a la violencia de pareja. El abordaje de estos problemas es reciente en lo que refiere a investigación.

De acuerdo a los hallazgos, la población de victimas de VP se comporta de la misma manera que la población estudiada en las dos encuestas previas realizadas en el país en relación a la distribución de tipos de violencia, reflejando el mismo orden de frecuencias con la diferencia que el tercer lugar lo ocupa la patrimonial.

Acorde a la bibliografía consultada, el estudio muestra una alta tasa de depresión entre las mujeres que sufren violencia de pareja. En el mundo, diversas investigaciones observan que la depresión junto a los trastornos de ansiedad son los dos problemas de salud mental más frecuentes entre las víctimas mujeres (Amor, Echeburúa, de Corral, Zubizarreta, \& Sarasua, 2002; Plazaola-Castaño, 2004). Los datos sugieren que la violencia de pareja es muy probablemente uno de los factores que inciden en la aparición de depresión. Por un lado se observa que la 
frecuencia de depresión en víctimas de VP es significativamente superior a la encontrada en personas no expuestas a VP. A ello se suma que se aislaron de la muestra otras causas frecuentes de depresión (duelos por personas queridas ocurridos en el último año, enfermedades crónicas -diabetes e hipertensión arterial-, enfermedades endócrinas y cardíacas, dolores crónicos y/o inhabilitantes y limitaciones funcionales -sordera, hipoacusia, dificultades motoras, etcétera-). De este modo, si bien se vería reducido el $n$ de la muestra, se consiguió que los resultados se aproximaran mejor a magnitudes de depresión e ideación suicida más asociadas a la VP. Hay que señalar que no se aislaron otros factores de riesgo para sintomatología depresiva e ideación suicida como ser otros duelos, problemas laborales y económicos, uso problemático de sustancias, cáncer, VIH y otras formas de violencia concurrentes con la de pareja (Campagne, 2012).

Otro factor que, de acuerdo a la literatura, incide en la aparición de sintomatología depresiva es el embarazo y el puerperio (Jadresic, 2010). En este estudio dicha variable fue registrada en la recolección de datos para analizar su comportamiento en relación con la depresión y la ideación suicida. Los datos indican que, en esta muestra, el embarazo no se asocia con los puntajes de depresión tanto en casos o controles. Este hallazgo permite sugerir entonces que la asociación significativa encontrada entre violencia y depresión no está influenciada por el embarazo como factor de riesgo para depresión.

El hecho de que los puntajes totales de depresión en las víctimas de VP sean significativamente más altos que en los controles muestra que, además de una mayor frecuencia de depresión en las víctimas, la magnitud o intensidad de la misma es superior en dicha población frente a las mujeres que no sufren VP.

La frecuencia de Ideación Suicida reportada por las mujeres que sufrieron violencia de pareja al contestar la Primera encuesta... (Inmujeres-MIDES, 2013) es similar a la recogida en nuestro estudio: $21,6 \%$ y $20 \%$ respectivamente. Esta frecuencia también 
es semejante a la reportada por otras investigaciones que han empleado los mismos instrumentos usados en este estudio (Velarde Ruiz, 2015). En el estudio que aquí se informa la frecuencia de IS es casi seis veces mayor en los casos de VP que en los controles (no VP) y la diferencia tiende a la significación estadística. Es posible que ésta no se alcance por tratarse de una muestra pequeña. En cualquier caso, es un aspecto a seguir investigando con muestras con similares criterios de exclusión pero con un $\mathrm{N}$ mayor.

En cuanto a la combinación de depresión e Ideación Suicida, cabe resaltar que todos los casos clínicamente severos de depresión, es decir que presentan ideación suicida, se encuentran entre la población expuesta a violencia de pareja. Este hecho fue observado también por Vásquez Machado (2007) quien determinó, en un estudio comparativo de mujeres deprimidas maltratadas y no maltratadas por sus parejas, que la ideación suicida es más frecuente entre las deprimidas maltratadas.

Desde un enfoque de riesgo, el $17 \%$ de depresión+ ideación suicida encontrado en el grupo de casos de VP pauta que casi 1 de cada 5 mujeres víctimas de violencia de pareja presentan, además de este factor de riesgo para el suicidio, otros dos factores principales, depresión e ideación suicida, lo que contrasta con población no expuesta a VP. Dada esta elevada frecuencia, se plantea la necesidad de explorar estos problemas en las consultas y controles en el primer nivel de atención, como estrategia de prevención de otras conductas suicidas más graves como el intento de suicidio o el suicidio consumado.

Considerando la asociación significativa entre la presencia de depresión, ideación suicida o depresión + ideación suicida y el estado civil, especialmente en los casos de VP, es necesario abordar el problema que esta muestra tiene con relación a la diferencia de distribución de esta última variable en ambos grupos, para una mejor comprensión de las diferencias significativas entre víctimas y no victimas de VP en cada una de las variables independientes estudiadas. 
Por un lado, el hecho que las solteras, separadas y divorciadas, que son quienes presentan una frecuencia significativamente superior de depresión, ideación suicida o ambos fenómenos combinados, estén sobre representadas en el grupo de víctimas de VP, plantea el problema de si se trata de un sesgo de selección o refleja un mayor índice de separación debido a la violencia de pareja. Esta es una interrogante a explorar en futuros estudios sobre la problemática.

Por otro lado, conociendo que en nuestro estudio la violencia de pareja es un factor de riesgo para la depresión y que en el grupo de no violencia (control) están sobrerepresentadas las casadas+unión libre, debemos relativizar este resultado. Debe ser éste un aspecto a continuar estudiando ya que también existen en la literatura consultada controversias sobre la incidencia del estado civil en la depresión con poblaciones de víctimas de violencia doméstica y de pareja. Según investigaciones con distintas poblaciones donde se estudió la depresión en expuestas a VP, se ha visto que es más frecuente la depresión entre quienes están solteras o separadas (Griboski, Guilhem \& Moura, 2015). También se informa de estudios en los que las casadas o en unión libre víctimas de violencia de pareja presentan mayor frecuencia de depresión (Guedes, García-Moreno \& Bott, 2014). Esto abre la interrogante sobre cómo se comportaría la variable depresión si se emparejan ambos grupos (casos y controles) por estado civil, incorporando al grupo control más casos de separadas, solteras o divorciadas. En el caso de la ideación suicida o de depresión + ideación suicida también es preciso insistir en que el comportamiento de la variable estado civil debe ser estudiado con más profundidad en otras instancias por los aspectos ya señalados con relación a la muestra.

Así como se excluyeron de la muestra otras causas de sintomatología depresiva, los casos de embarazo y puerperio fueron admitidos ya que el estudio se realizó en policlínicas de Ginecobstetricia (además de otras) y la población participante del estudio se vería más limitada. También se consideró estratégicamente útil su inclusión 
ya que el estudio pretende investigar estos problemas entre la población femenina que asiste al primer nivel de atención, dentro de la cual el control del embarazo y puerperio están muy presentes en las consultas. Dado que cursar o no cursar un embarazo en el momento del estudio no arroja diferencias significativas en la presencia de depresión, los resultados sugieren que aún pudiendo ser causa de sintomatología depresiva, no explica la aparición de depresión en los casos de violencia de pareja. Esto es muy importante desde el punto de vista del análisis correlacional entre la violencia y los problemas de salud mental estudiados aquí ya que la violencia de pareja correlaciona más claramente con la depresión y la ideación suicida que el embarazo.

Asimismo, nuestros datos mostraron que el tiempo transcurrido desde la exposición a la violencia no se asocia con la presencia de depresión, Ideación Suicida o Depresión e Ideación Suicida combinados. Si bien se han documentado estudios en los que se determina que haber padecido recientemente violencia de pareja aumenta considerablemente el riesgo suicida y particularmente los intentos de autoeliminación (Olshen, McVeigh, Wunsch-Hitzig \& Rickert, 2007) no identificamos aún estudios con los que podamos discutir la comparación entre la violencia actual y pasada y su relación con el riesgo suicida.

Finalmente, es posible que sea útil replicar estudios como éste en centros de salud y policlínicas del primer nivel de atención, considerando que la utilización de estos instrumentos de evaluación psicológica y de una entrevista clínica estructurada enfocada a la detección y caracterización de la violencia de pareja ha mostrado ser una batería de técnicas sencilla y eficaz para aplicar en estos contextos. Aunque no fuera parte de la investigación, es destacable que se haya observado que la gran mayoría de los casos de VP no habían sido captados por los equipos de salud así como tampoco los de depresión ni los de conducta suicida. En este sentido se aportaría a la detección precoz de la violencia de pareja y a la evaluación y detección precoz de algunos de los principales problemas de salud mental vinculados a dicha 
problemática, especialmente como prevención del intento de autoeliminación y el suicidio. Esto es particularmente relevante sabiendo que en Uruguay los prestadores de salud pública y privada cuentan con equipos de referencia de violencia doméstica para asegurar la atención de esta problemática una vez detectada. Los datos estarían poniendo de relieve que estos recursos tiene aún una cobertura insuficiente por fallas en la captación de las víctimas y de algunos problemas asociados.

\section{Referencias}

American Psychiatric Association (2013). Diagnostic and Statistical Manual of Mental Disorders (5a ed.). Arlington: American Psychiatric Association.

Amor, P. J., Echeburúa, E., de Corral, P., Zubizarreta, I. \& Sarasua, B. (2002). Repercusiones psicopatológicas de la violencia doméstica en la mujer en función de las circunstancias del maltrato. Revista Internacional de Psicología Clínica y de la Salud. 2(2), 227-246. Recuperado de http://www.aepc.es/ijchp/articulos_pdf/ijchp-38.pdf

Beck, A.T., Steer, R. A. \& Brown, G. K. (2011). Inventario de Depresión de Beck-II. Adaptación española: Sanz, J \& Vázquez, C. Madrid: Pearson Educación.

Brenlla, M.E. \& Rodríguez, M. (2006). Adaptación Argentina del Inventario de Depresión de Beck-II (BDI-II). En A.T. Beck, R.A. Steer \& G.K. Brown (Eds.), BDI-II. Inventario de Depresión de Beck (pp. 11-37). Buenos Aires: Paidós.

Campagne, D. M. (2012). Causas orgánicas y comórbidas de la depresión: el primer paso. Semergen. 38(5), 301-311. Recuperado de https://medes.com/publication/75368

Canetti, A. (2017). La conducta suicida desde la perspectiva psiquiátrica. Una revisión de la situación en Uruguay. En Larrobla, C. et al (Comp.), 70 años de suicidio en Uruguay: 7 disciplinas, 7 entrevistas, 7 encuentros (pp. 107-134). Montevideo: CSIC-Universidad de la República. 
Canetti, A., Larrobla, C, Reigia, M \& Brenes, V. (2011). Sociocultural representation about attempts of suicide in adolescents: a students and educators vision. Abstracts 15th World Congress of Psychiatry [CD-ROM]. Buenos Aires: World Psychiatric Association.

Devries, K.M., Mak, J. Y., Bacchus, L. J., Child, J.C., Falder, G., Petzold, M., \& Watts, CH. H. (2013). Intimate Partner Violence and Incident Depressive Symptoms and Suicide Attempts: A Systematic Review of Longitudinal Studies. PLoS Med 10(5): e1001439. doi:10.1371/journal.pmed.1001439

Fogarty, C.T. \& Brown, J. B. (2002). Screening for abuse in spanish-speaking women. Journal of the American Board of Family Practice. 15, 101-111. Recuperado de http://www.jabfm.org/content/15/2/101.full.pdf+html

Gibbons, L. (2011). Dealing with the effects of domestic violence. Emergency Nurse, 19(4),12-6;quiz17.

Recuperado de: https://www.ncbi.nlm.nih.gov/pubmed/21877612

Griboski, R., Guilhem, D., \& Moura, L. (2015). Occurrence of violence caused by intimate partners in brazilian rural working women: a descriptive study. Online Brazilian Journal of Nursing, 14(2), 151-60. doi:http://dx.doi.org/10.17665/16764285.20154653

Guedes, A., García-Moreno, C. \& Bott, S. (2014). Violencia contra las mujeres en Latinoamérica y el Caribe. Foreign Affairs Latinoamérica. 14(1), 41-48. Recuperado de www.fal.itam.mx

Inmujeres-MIDES. (2013). Primera Encuesta Nacional de Prevalencia Sobre Violencia Basada en Género y Generaciones, año 2013. Informe de resultados (2013). Montevideo: Inmujeres-MIDES Recuperado de http://www.inmujeres.gub.uy/innovaportal/file/33876/1/resumen_de_encuesta_ mides.pdf

Jadresic M, E. (2010). Depresión en el embarazo y el puerperio. Revista chilena de 
neuro-psiquiatría, 48(4), 269-278. doi:https://dx.doi.org/10.4067/S0717$92272010000500003019-1028$

Lozano, F, Salas Rosso, F \& Paullier, A (2011). Descubrir la violencia doméstica en la consulta médica para trascender el tratamiento sintomático. Revista $\begin{array}{llll}\text { Biomedicina, } & 6(2), & \text { 16-28. } & \text { Recuperado de }\end{array}$ http://www.um.edu.uy/docs/violencia_domestica.pdf

Ministerio de Salud Pública, Facultad de Medicina, \& Instituto Nacional de Estadística. (2013). Encuesta de Prevalencia de Violencia Doméstica entre mujeres mayores de 15 años asistidas en servicios de salud pública y Privada. Principales Resultados. Montevideo: Uruguay. Recuperado de http://www.msp.gub.uy/sites/default/files/archivos_adjuntos/Binder1.pdf

Olshen, E., McVeigh, K., Wunsch-Hitzig, R. A., \& Rickert, V.I. (2007). Dating Violence, Sexual Assault, and Suicide Attempts Among Urban Teenagers. Archives of Pediatrics an Adolescent Medicine, 161(6), 539-545. Recuperado de https://www.ncbi.nlm.nih.gov/pubmed/17548757

Organización Mundial de la Salud [OMS]. (2005). Estudio multipaís de la OMS sobre salud de la mujer y violencia doméstica contra la mujer: primeros resultados sobre prevalencia, eventos relativos a la salud y respuestas de las mujeres a dicha violencia. Resumen del Informe. Ginebra: Organización Mundial de la Salud.

Organización Panamericana de la Salud [OPS]. (2009). Estrategia y plan de acción sobre salud mental. Washington, D.C.: Organización Panamericana de la Salud, Oficina Regional para las Américas de la Organización Mundial de la Salud.

PDM Task Force (2006). Psycodinamic Diagnostic Manual. Silver Spring, MD: Alliance of Psychoanalytic Organizations 
Plazaola-Castaño, J., Ruiz-Pérez, I. \& Hernández-Torres, E. (2008). Validación de la versión corta del Woman Abuse Screening Tool para su uso en atención primaria en España. Gaceta Sanitaria, 22(5), 415-420.

Plazaola-Castaño, J., \& Ruiz Pérez, I. (2004). Violencia contra la mujer en la pareja y consecuencias en la salud física y psíquica. Medicina Clínica, 122(12), 461467. doi: https://doi.org/10.1016/S0025-7753(04)74273-6

Romano, S. (2010). Violencia de género en la pareja. Aportes para su evaluación en la consulta psiquiátrica de mujeres. Revista de Psiquiatría del Uruguay, 74(1), 4566.

Recuperado

de http://www.spu.org.uy/revista/ago2010/05_VIOLENCIA\%20DE\%20GENERO.pd f

Vásquez Machado, A. (2007). Relación entre violencia y depresión en mujeres. Revista de Neuro-Psiquiatría, $70 \quad(1-4)$ 88-95. Recuperado de http://www.redalyc.org/pdf/3720/372039390004.pdf

Velarde Ruiz, R. Y. (2015). Violencia de pareja e ideación suicida en mujeres de la Institución Educativa Adventista 28 de Julio de la Ciudad de Tacna (Tesis de maestría). Universidad Peruana Unión, Lima. Recuperado de http://repositorio.upeu.edu.pe/handle/UPEU/216

\section{Declaración de contribución de los autores}

SLI participó en el diseño e implementación de la investigación y en la escritura del manuscrito. AC colaboró en el procesamiento, análisis de los resultados y escritura del trabajo. Ambos autores discutieron los resultados y contribuyeron a la versión final del manuscrito. 
Formato de citación

Llosa, S. y Canetti, A. (2018). Depresión e ideación suicida en mujeres víctimas de violencia de pareja. Psicología, Conocimiento y Sociedad, Preprint, 1-27. Disponible en: http://revista.psico.edu.uy/ 